\title{
Nonlinear System Identification Method based on Multi-dimensional Taylor Network and its Applications in Construction Safety Monitoring
}

\author{
Yi Lin ${ }^{1,2 \mathrm{a}}$, Yifei Chen ${ }^{1,2 \mathrm{~b}}$, Liling Zhao ${ }^{1,2 \mathrm{c}}$ and Yufang Wang ${ }^{1,2 \mathrm{~d}}$
}

${ }^{1}$ School of Information and Control, Nanjing University of Information Science \& Technology, Nanjing, China

2 Jiangsu Key Laboratory of Big Data Analysis Technology, Nanjing University of Information Science \& Technology, Nanjing, China

anuistly@163.com, ${ }^{b}$ ch_yi_f@126.com, c ring_222@126.com, ${ }^{\text {d }}$ qing_0325@163.com

Keywords: Nonlinear System Identification, Multi-dimensional Taylor Network, Safety Monitoring, Prediction

\begin{abstract}
A novel nonlinear system identification method based on the Multi-dimensional Taylor Network is proposed in this paper. It provides a new method to identify the nonlinear system, which can describe the dynamic characteristics without prior knowledge and can realize the prediction of the nonlinear time series just with input-output data. Firstly, the structure of the Multi-dimensional Taylor Network is introduced. Then, a dynamic model based on the Multi-dimensional Taylor Network is established. Finally, this method is applied in construction safety monitoring to predict the vibration response of building structures. Results from the simulation example demonstrate the effectiveness and feasibility of the proposed method.
\end{abstract}

\section{Introduction}

The nonlinear system identification is widely applied in many fields. The world consists of a variety of systems and the models of the systems can be got by identification. However, it is difficult to get the models of every system based on the clear internal mechanism. On the one hand, the mechanism of most systems is partially known or unknown at all, and on the other hand, the models of complex nonlinear systems with known structures are difficult to achieve due to too many internal factors. Therefore, it is necessary to find other solutions to solve the problem [1]. Artificial neural network and the support vector machine have been widely applied in modeling and prediction, and the research results are plentiful. But there are some disadvantages such as limited approximation ability and the determination of the kernel [2-5].

A novel nonlinear system identification method based on the multi-dimensional Taylor network is presented in this paper. Without the clear internal mechanism and structure, the multi-dimensional Taylor network dynamics model can be obtained which can describe the dynamic characteristics of the nonlinear system just based on the input-output data. The identification method has been applied in lifting construction safety monitoring to predict the vibration response of building structures and results from the simulation example demonstrate the effectiveness and feasibility of the proposed method.

\section{Construction of the model}

General dynamic equations of the system can be written as

$\left\{\begin{array}{l}\boldsymbol{x}(k+1)=\boldsymbol{f}(\boldsymbol{x}(k))+\boldsymbol{u}(\boldsymbol{x}(k)) \\ \boldsymbol{y}(k)=\boldsymbol{g}(\boldsymbol{x}(k))\end{array}\right.$

where $\boldsymbol{x}$ is the system state vector, and $\boldsymbol{y}$ is the system output vector; nonlinear function $\boldsymbol{f}$ is equivalent to $\boldsymbol{x}$ 's dynamic mapping; $\boldsymbol{u}$ is equivalent to the system state mapping; function $\boldsymbol{g}$ represents the state-output mapping. Ignoring the mappings between state control vectors and focusing solely on the system dynamic mappings of state vector $\boldsymbol{x}$ simplify Eq.(1) as below: 


$$
\left\{\begin{aligned}
\boldsymbol{x}(k+1) & =\boldsymbol{f}(\boldsymbol{x}(k)) \\
\boldsymbol{y}(k) & =\boldsymbol{g}(\boldsymbol{x}(k))
\end{aligned}\right.
$$

where $\boldsymbol{x}$ is the system state vector, $\boldsymbol{y}$ is the system output vector, $\boldsymbol{g}$ is state-output mapping vector function, and $\boldsymbol{f}$ is the nonlinear vector function. The explicit form of the non-linear function $f$ in the first line of Eq. (1) can be expressed by multidimensional Taylor network model.

For an $n$-dimensional system, the first line of Eq. (3) can be rewritten in the scalar form, i.e.,

$$
\left\{\begin{array}{c}
x_{1}(k+1)=f_{1}\left(x_{1}(k), x_{2}(k), \cdots, x_{n}(k)\right) \\
x_{2}(k+1)=f_{2}\left(x_{1}(k), x_{2}(k), \cdots, x_{n}(k)\right) \\
\vdots \\
x_{n}(k+1)=f_{n}\left(x_{1}(k), x_{2}(k), \cdots, x_{n}(k)\right)
\end{array} .\right.
$$

where the nonlinear function $f_{j}$ is equivalent to the $x_{1}(k) \sim x_{n}(k)$ 's dynamic mapping. In terms of the model structure and its definition of multidimensional Taylor network, $f_{j}$ can be constructed as

$$
f_{j}\left(x_{1}, x_{2}, \cdots, x_{n}\right)=\sum_{q=1}^{w} \lambda_{j, q} \prod_{i=1}^{n} x_{i}^{\sigma_{q, i}}+R_{j, m}\left(x_{1}, x_{2}, \cdots, x_{n}\right), \quad j=1,2, \cdots, n
$$

s.t. $\quad \sum_{i=1}^{n} \sigma_{q, i} \leq m$,

where $R_{j, m}$ is the sum of the remaining items, each being a product of the variables whose sum of powers is greater than $m ; \lambda_{j, q}$ is the $q$-th weight before each item; $\sigma_{q, i}$ is the power of the variable $x_{i}$ in the $q$-th product term of the variables.

The multi-dimensional Taylor network model is given in Fig. 1. The input is $\boldsymbol{x}(k)$ which can be defined as $\boldsymbol{x}(k)=\left[x_{1}(k), x_{2}(k), \cdots, x_{n}(k)\right]^{\mathrm{T}} \in R^{n}$. The hidden layer is consisted of power function product terms and the weights connecting the function product terms to the output units. The hidden layer is used to get the weighted sum of each power function product terms, which is composed of power function product terms and the corresponding weight vector $\lambda_{l}$ which is the vector between the hidden layer nodes and the output. The output is $\boldsymbol{x}(k+1)$, which can be defined as $\boldsymbol{x}(k+1)=\left[x_{1}(k+1), x_{2}(k+1), \cdots, x_{n}(k+1)\right]^{\mathrm{T}} \in R^{n}$.

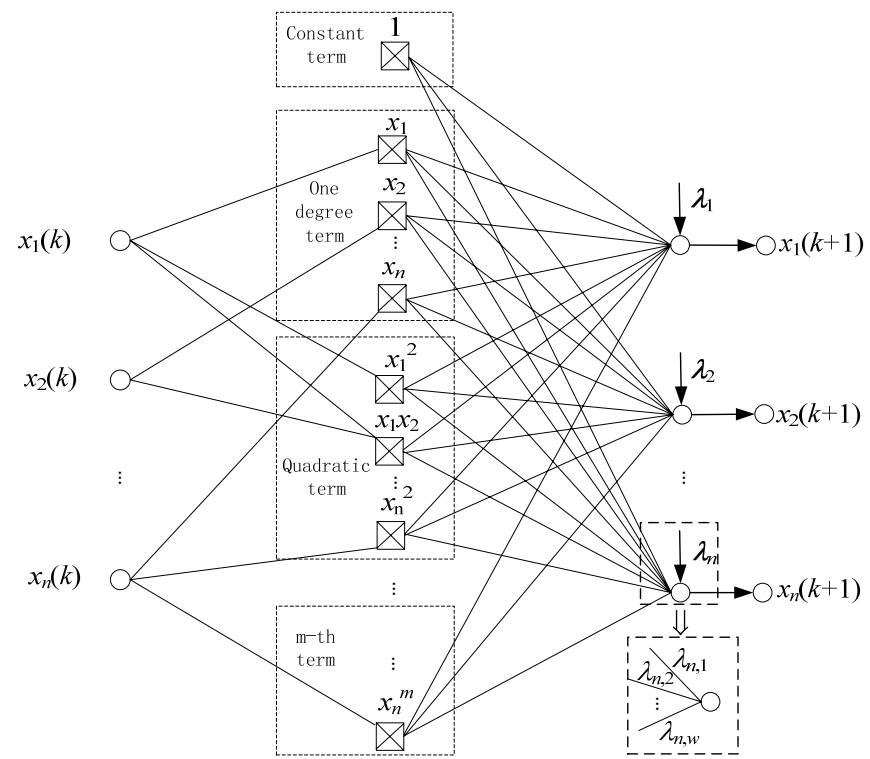

Fig.1 Multi- dimensional Taylor Network model 
With the gradual increase of state vector $x_{i}^{\prime}$ s power sum, weight of product items drops. So when selecting a suitable power sum of $m, R_{j, m}$ items can be ignored. For an $n$-dimensional system of maximum power $m$, the scalar equation can be expressed as

$$
\left\{\begin{array}{c}
x_{1}(k+1)=\sum_{q=1}^{w} \lambda_{1, q} \prod_{i=1}^{n} x_{i}^{\sigma_{q, i}}(k) \\
x_{2}(k+1)=\sum_{q=1}^{w} \lambda_{2, q} \prod_{i=1}^{n} x_{i}^{\sigma_{q, i}}(k) \\
\vdots \\
x_{n}(k+1)=\sum_{q=1}^{w} \lambda_{n, q} \prod_{i=1}^{n} x_{i}^{\sigma_{q, i}}(k)
\end{array}\right.
$$

the scalar equation can describe the dynamic characteristics without prior knowledge and can realize the prediction of the nonlinear system just with input-output data.

\section{Examples of the application in construction safety monitoring}

Lifting is a special construction technique in structural modification and during the construction, vibration response is monitored in real time so as to ensure the structural safety. If the next moment of structural vibration response can be forecasted accurately in advance and corresponding response can be made ahead of time, the possibility of structural damage is greatly reduced.

The experimental model is a three-layer frame structure. The position of the sensors is shown in figure 2 and these sensors are set to monitor the real-time dynamic response of the main structure in the jacking process. The sensor with number $\mathrm{H}$ indicates the vibration of the horizontal direction and the sensors with number $\mathrm{V}$ indicates the vibration of the vertical direction. Take the vibration data of the vertical direction measured by the sensor in the third layer structure as example. We applied the proposed method in this paper to forcast the next moment of structural vibration response in order to monitor the safety of the structure in advance.

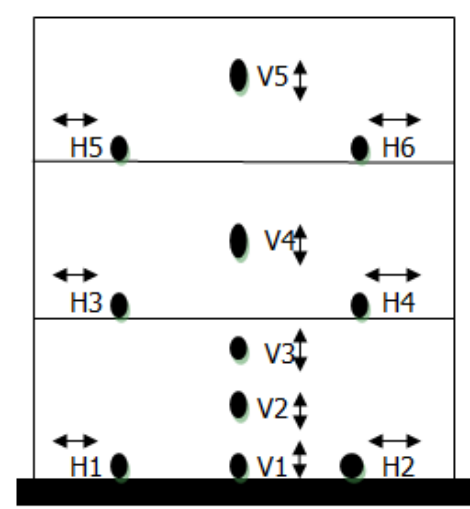

Fig.2 diagram of sensors position

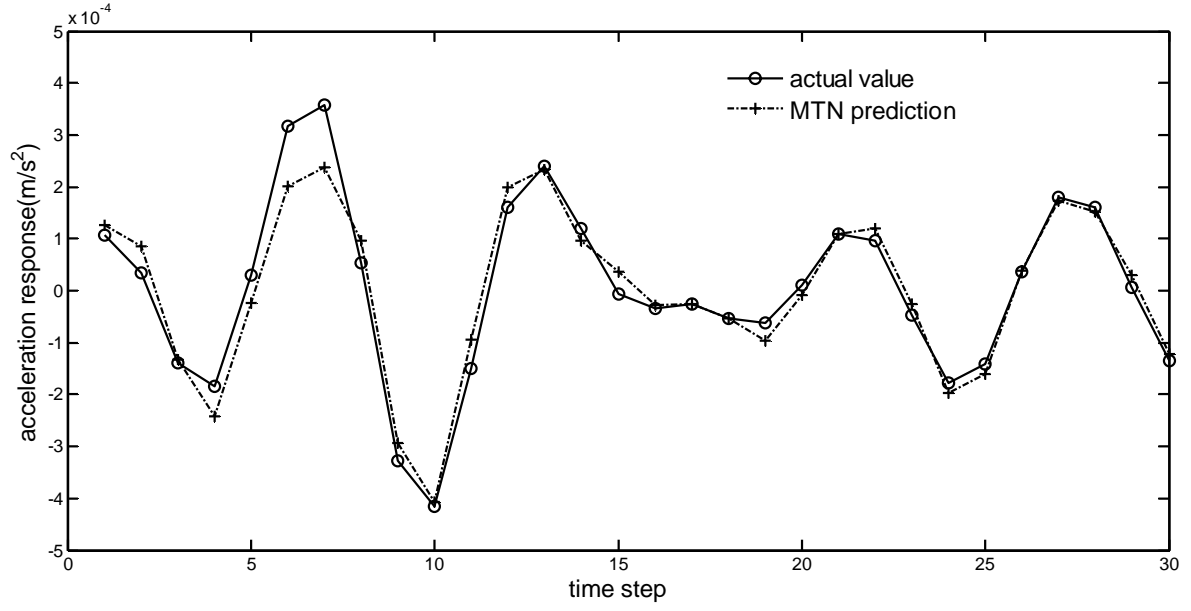

Fig.3 Prediction based on the multi-dimensional Taylor network

Firstly, the model based on the multi-dimensional Taylor network is built. The parameters are identified on the basis of the training set of 100 sample data. Then, 30 prediction values of the trained model are derived from 30 test data and the test data is to verify the performance of the method. Actual values and predicted values are shown as Fig. 3. Seen from the Fig.3, it indicates that the prediction accuracy of this method is satisfactory.

The error indictors of MAE (Mean Absolute Error) and RMSE (Root Mean Square Error) are used to evaluate the predictive precision. The MAE and RMSE are defined as:

MAE $=\frac{1}{N} \sum_{t=1}^{N}|y(k+1)-\hat{y}(k+1)|$ 


$$
\text { RMSE }=\sqrt{\frac{1}{N} \sum_{t=1}^{N}[y(t+1)-\hat{y}(t+1)]^{2}}
$$

where $N$ represent the number of the test sample, $y(t+1)$ represent the real value, $\hat{y}(t+1)$ represent the prediction.

Neural networks are widely used in time series prediction. The BP neural network and RBF-BP combined neural network are used to predict the same time series in order to discuss the performance of proposed method. Comparison results are presented in Table 1.

Table.1 Comparison of error indicators

\begin{tabular}{ccc}
\hline Model & MAE & RMSE \\
\hline MTN & 2.94 E-05 & 4.16 E-05 \\
BP & 4.20 E-05 & 5.38 E-05 \\
RBF-BP & $3.12 \mathrm{E}-05$ & $4.56 \mathrm{E}-05$ \\
\hline
\end{tabular}

As can be seen, indicators of the MTN model are better than the BP model and RBF-BP model. It demonstrates the validity and accuracy of the proposed model.

\section{Conclusions}

A new approach to dynamic system modeling based on the Multi-dimensional Taylor Network has been proposed and testified in this paper. It can get the dynamics scalar equation of the nonlinear system just with the input and output data and without any prior knowledge and mechanism of the system. This new system identification method can be used to predict time series. Take the acceleration response prediction in safety monitoring of lifting construction as example and the results demonstrate that the MTN approach guarantee a higher prediction accuracy than that the other two models based on the neural network.

\section{Acknowledgement}

This work is supported by the program of the National Natural Science Foundation of China under Grant 61573193.

\section{References}

[1] Cholette M E, Liu J B, Djurdjanovic D, et al. Monitoring of complex systems of interacting dynamic systems[J]. Applied Intelligence, Vol. 37 (1) (2012), p. 60-79.

[2] Y. Li, L.P. Liu, B.Q. Li, et al. Calculation of Line Loss Rate in Transformer District Based on Improved K-Means Clustering Algorithm and BP Neural Network[J]. Proceedings of the CSEE, Vol. 36 (17) (2016), p. 1581-1585.

[3] F. Ahmadizar, K. Soltanian, F. AkhlaghianTab, et al. Artificial neural network development by means of a novel combination of grammatical evolution and genetic algorithm[J]. Engineering Applications of Artificial Intelligence, Vol. 39 (2015), p. 1-13.

[4] Z.H. Dai, X.G. Ren, G. Shu. Prediction of wind power by chaos and BP artificial neural networks approach based on genetic algorithm[J]. Journal of Electrical Engineering \& Technology, Vol. 10(1) ( 2015), p. 41-46.

[5] Q. Wu, L.Y. Zhang, W. Wang. New family of piecewise smooth support vector machine[J]. Journal of System Engineering and Electronics, Vol. 26(3) (2015), p. 618-625. 